\title{
An Introductory Note on Finite Element Problems Based on the Eddy Current Testing Approach
}

\author{
Antônio Flavio Licarião Nogueira*, Rodolfo Lauro Weinert, \\ Leonardo José Amador Salas Maldonado \\ Department of Electric Engineering, Santa Catarina State University, Joinville, Brazil \\ Email: *antonioflavio.licariaonogueira@gmail.com
}

How to cite this paper: Nogueira, A.F.L., Weinert, R.L. and Maldonado, L.J.A.S. (2021) An Introductory Note on Finite Element Problems Based on the Eddy Current Testing Approach. Journal of Electromagnetic Analysis and Applications, 13, 145-159.

https://doi.org/10.4236/jemaa.2021.1311011

Received: September 3, 2021

Accepted: October 31, 2021

Published: November 3, 2021

Copyright $\odot 2021$ by author(s) and Scientific Research Publishing Inc. This work is licensed under the Creative

Commons Attribution International License (CC BY 4.0).

http://creativecommons.org/licenses/by/4.0/

\begin{abstract}
The paper addresses the first eddy current benchmark problem proposed by the World Federation of Nondestructive Evaluation Centers (WFNDEC). The problem simulates the eddy current response to the presence of an axisymmetric circumferential defect in an Inconel-600 tube. All simulations employ the axisymmetric code of the electromagnetic field simulator Finite Element Method Magnetics. For three different frequencies of excitation, it is explained how the displacement of the detecting coil inside the tube leads to a variation in the impedance of the eddy current coil. Variations of the resistive and inductive components of the impedance with distance from the defect region are used to build the impedance trajectory for each frequency of analysis.
\end{abstract}

\section{Keywords}

Eddy Current Testing, Finite Element Analysis, Nondestructive Inspection Methods

\section{Introduction}

The first eddy current benchmark problem proposed by the World Federation of Nondestructive Evaluation Centers (WFNDEC) simulates the eddy current response to the presence of an axisymmetric circumferential defect in an Inconel-600 tube [1]. A cross-sectional view of the problem is shown in Figure 1, and the geometrical dimensions are presented in Table 1. The simulations have been carried out using the two-dimensional axisymmetric code of the electromagnetic field simulator Finite Element Method Magnetics (FEMM) [2]. All 
Table 1. Geometrical dimensions for the WFNDEC' $1^{\text {st }}$ eddy current problem.

\begin{tabular}{cc}
\hline Parameter & Length $(\mathrm{mm})$ \\
\hline$h_{1}$ & 0.2 \\
$h_{2}$ & 0.3 \\
$h_{3}$ & $0,0.1,0.5,1.0$ \\
$h_{4}$ & 2.0 \\
$d$ & 20.0 \\
$D_{1}$ & 19.7 \\
$D_{2}$ & 22.24 \\
$D_{3}$ & 9.0 \\
$D_{4}$ & 19.0 \\
$D_{5}$ & $22.24,23.24,24.24$ \\
$D_{6}$ & 100.0 \\
\end{tabular}

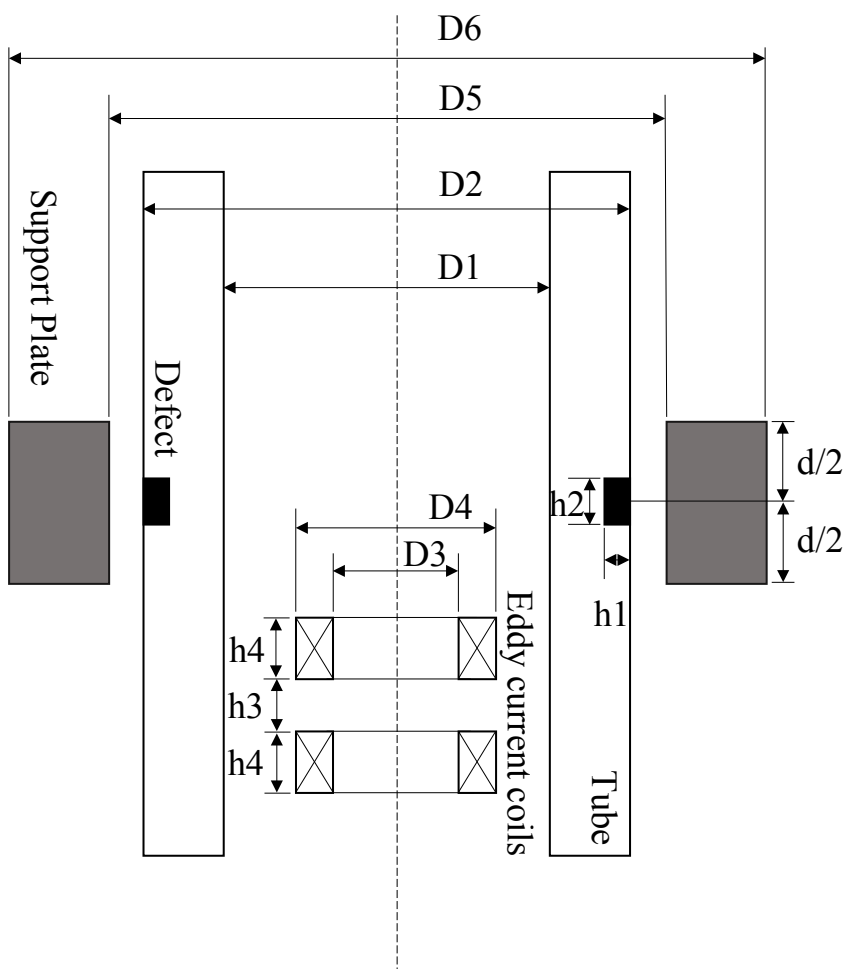

Figure 1. Cross-sectional view of the WFNDEC' $1^{\text {st }}$ eddy current problem.

simulations employ the linear time-harmonic technique, and the finite element code employs the $\boldsymbol{A}$ - $V$ formulation with the electric scalar potential $V$ set to zero [3].

Eddy current testing (ECT) is a computational method with many industrial applications including crack detection to the rapid sorting of small components for flaws, size variations as well as material variations. The impedance of the detecting coil is distorted and altered by the presence of flaws or material varia- 
tions [5]. Also, the dissipated power in the conductive region containing the flaw is altered. The calculation of eddy current coil impedances in the $A$ - $V$ formulation is discussed in Appendix A. ECT is one of the nondestructive evaluation (NDE) data fusion techniques. Nondestructive inspection methods also include techniques like ultrasound, optical, thermography and radiography. One of the earliest review papers on nondestructive evaluation (NDE) data fusion techniques has been presented by Gros, in 1997 [5]. The widespread use of NDE fusion techniques has initially been registered on the book of Gros [6] and later, at the World Conferences on NDE hosted in Rome and Montreal in the years of 2000 and 2004, respectively [7] [8].

As it can be observed in Figure 1 and Table 1, the configuration of the test problem changes by varying the axial distance between the two coils (parameter $h_{3}$ ) and/or the gap between the tube and support plate (parameter $D_{5}$ ). In the configuration selected for the simulated work, the gap between the two coils is $h_{3}$ $=0.5 \mathrm{~mm}$, and the gap between the tube and support plate is also $0.5 \mathrm{~mm}$. These choices appear highlighted in Table 1. Additional choices include material properties 1) the electrical conductivities, $\sigma$, of the Inconel-600 tube and support plate are both equal to $1.0 \times 10^{6} \mathrm{~S} / \mathrm{m}$; and 2) the magnetic relative permeability, $\mu_{\mathrm{r}}$, of the support plate is 100. In all simulated problems, two identical air-cored coils with 1000 turns each are connected differentially and used as eddy current excitation and detection system. For short, the pair of air-cored coils will be referred to as eddy current coil. In the sequence of simulations, the eddy current coil moves downwards inside the Inconel-600 tube in the axial direction. The analysis includes three frequencies of excitation: 1, 10, and 100 kilohertz.

Results of the WFNDEC's first benchmark problem have been published by different research groups. Initially, came the work of Sikora and Palka in 2001 [9] and later, the work of Tian et al. in 2004 [10], respectively. These research groups have used different ECT codes based on the magnetic vector-scalar potential $\boldsymbol{A}$ - $\boldsymbol{V}$ formulation [11] [12]. Data presented in these papers consist of impedance trajectories in the $R$ - $X$ plane for four different frequencies of excitation. Unfortunately, different research groups have used different parameters, and published results are not easily reproducible. The difficulties in reproducing the earlier published results presented in [9] and [10] are briefly discussed in the following.

In the WFNDEC's first benchmark problem, neither the material properties nor the physical arrangement of the wires that form the pair of coils is described. It is worth noting that the wires made of, say copper or aluminum and accommodated in a given coil region can be specified as 1) stranded wire; 2) not stranded wire; 3) magnet wire; 4) plain stranded wire; 5) litz wire; or 6) square wire. According to the authors' choice, each rectangular region that forms the pair of coils accommodates 1000-not stranded copper wires with unity relative permeability $\mu_{r}$ and electrical conductivity $\sigma=58 \times 10^{6} \mathrm{~S} / \mathrm{m}$. Also, the value of 10 $\mathrm{mA}$ mentioned in the problem description has been assumed to mean the root-mean-square (rms) value of the exciting current. Once the field simulator 
FEMM always works with "peak" values, in all simulated problems the terminal current of the eddy current coil is specified as $I_{\mathrm{p}}=14.142 \mathrm{~mA}$.

\section{Finite Element Model}

An outline of the axisymmetric model is shown in Figure 2. In this drawing, it is shown the main features of the field problem that represents the pair of coils symmetrically located with respect to the origin $(r=0 ; z=0)$ and defect area. According to the reference frame, at this position, the coil displacement $d$ is zero. To facilitate the visualization of the drawing, the size of the defect (flaw) is exhibited out of scale and enlarged more or less five times. The height of the Inconel-600 tube is $200 \mathrm{~mm}$. The external boundary that closes the domain of analysis is cylindrical, and its cross-section possesses a radius of $150 \mathrm{~mm}$ and a height of $300 \mathrm{~mm}$ symmetrically positioned about the origin $(r=0 ; z=0)$. The boundary conditions applied to the cylindrical boundary are truncation of the outer boundaries. The usual way of applying this mathematical condition is by prescribing $A=0$ at this boundary [13].

The grain of the finite element mesh in different regions of the model is defined by the parameter "edge size" [14]. This parameter defines a constraint on the largest possible size of the triangles' edges allowed in a given region. Smaller sizes for the edge size parameter have been specified in regions associated to larger amount of energy transfer and magnetic field inhomogeneity. It is the case, e.g., of the eddy current coil region and the air-gap between the tube and support plate. Special attention has been given to the meshing of the support plate's edges due to the presence of induced eddy currents. In the case, smaller

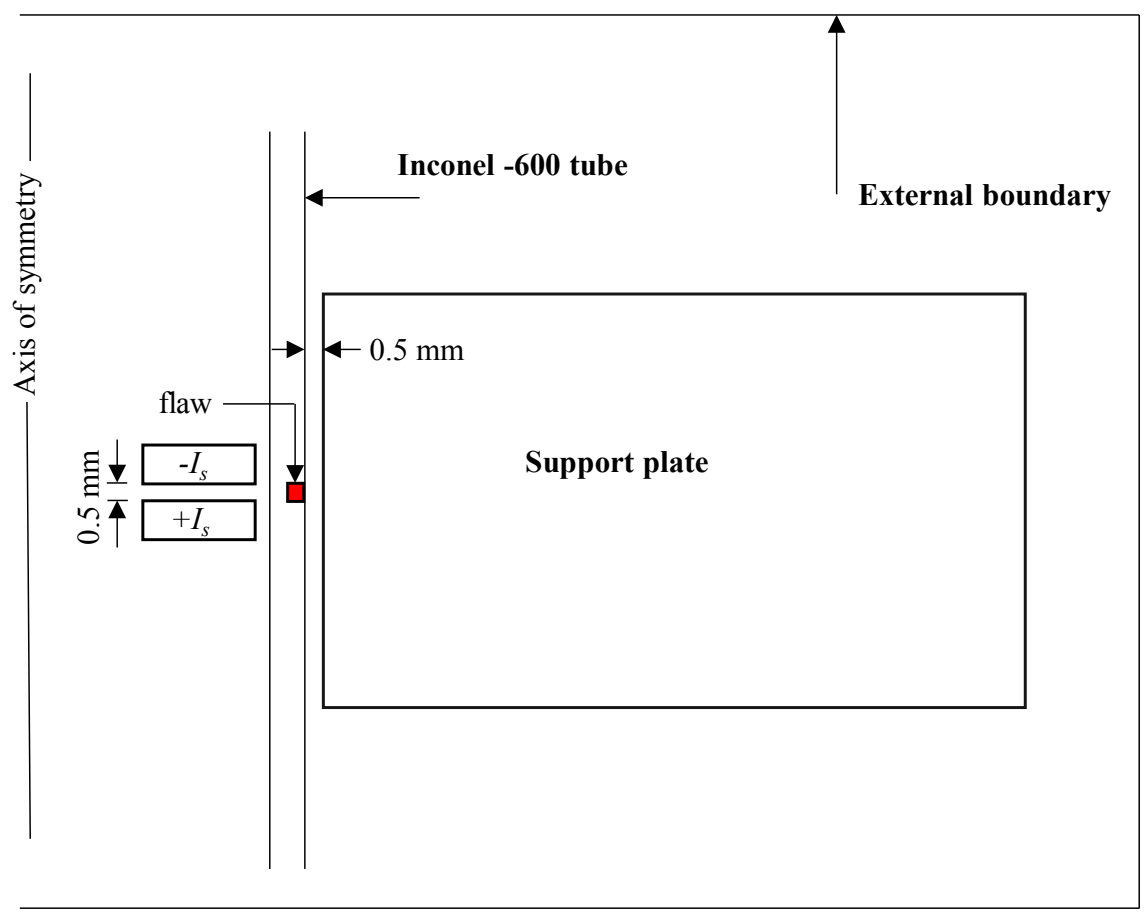

Figure 2. Outline of the axisymmetric model. 
values for the edge size parameter have been specified in regions such as the leftmost portion of the support plate near the air gap. As a result of these choices, economical meshes with an average of 65,000 nodes and 130,000 elements have been employed to save time and computer effort.

\section{Impedance Trajectories}

The impedance trajectory is plotted in the complex $R-X$ plane, and represents the variation of the eddy current coil impedance that occurs when the material medium of the region representing the defect (flaw) changes. To obtain the impedance trajectory associated to a given frequency of excitation, it is necessary to obtain two different sequences of magnetic vector potential solutions. The first sequence of vector potentials $\left\{\boldsymbol{A}_{1}\right\}$ provides the values of the coil impedance when the defect is present, i.e., the rectangular region of area $h_{1} \times h_{2}$ shown in Figure 1 is filled by air. The second sequence of vector potentials $\left\{\boldsymbol{A}_{2}\right\}$ provides the values of the coil impedance when the defect is not present, i.e., the rectangular region of area $h_{1} \times h_{2}$ is filled by the same material of the tube: Inconel-600. In both sequences, the displacement of the detecting coil, $d$, varies in the range $\left[0 ; d_{\max }\right]$. According to the reference frame, when the pair of coils is symmetrically positioned with respect to the origin and defect region, at $(r=0 ; z$ $=0), d=0$. When the detecting coil is sufficiently far away from the defect region, the difference $\Delta Z$ in the coil impedances given by the two sequences of vector potentials vanish. All additional generated points of the trajectory will be situated at the origin of the $R-X$ plane. The final shape of the correct or idealized impedance trajectory is a smooth, convex and closed loop. The distance $d=d_{\max }$ where $\Delta Z$ vanishes is problem-dependent, and varies with the frequency of excitation: for $10 \mathrm{kHz}, d_{\max }=-15 \mathrm{~mm}$, whilst for $100 \mathrm{kHz} d_{\max }=-11.75 \mathrm{~mm}$.

The coil movement is simulated employing steps of $0.25 \mathrm{~mm}$. The movement is the result of a sequence of vertical translations of the eddy current coil in the $-z$ direction. Each vertical translation requires a new mesh for the problem. This approach has been chosen because the artifice of material re-identification to change materials in problems representing consecutive positions of the pair of coils would require a very complicated drawing, with many distinct regions in the air layer that accommodates the eddy current coil.

\section{Simulations at 1.0 Kilohertz}

At this low level of operating frequency, the ECT approach is not reliable for this class of problem, and its use is quite rare. In the graph of Figure 3, two possibilities are considered 1) success in detecting variations in the coil impedance is indicated along the upper horizontal line; and 2) failure in detecting variations in the coil impedance is indicated along the lower horizontal line. A close observation of the illustration clearly shows that, along most of the excursion, the simulated experiment has not succeeded in detecting the impedance variations of the eddy current coil associated to the two sets of vector potential solutions. It is the 


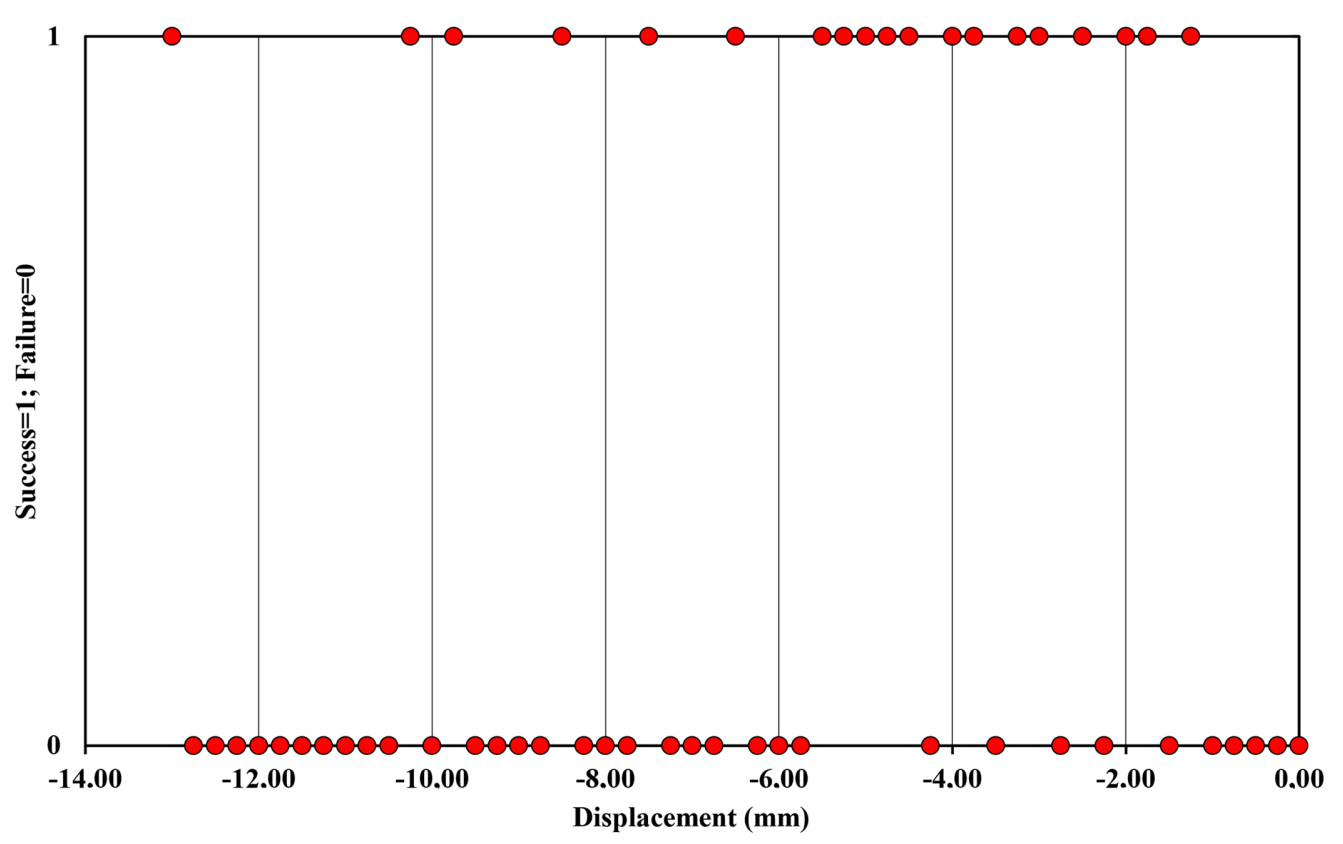

Figure 3. Detecting variations in the coil impedance at $1 \mathrm{kHz}$ : success (1); and failure (0).

case, for example, of the computations in the ranges $-12.75 \leq d \leq-10.50$ and $-9.50 \leq d \leq-8.75$. At this low level of operating frequency, the ECT approach is clearly questionable, perhaps even downright worthless for this class of problem.

\section{Simulations at 10.0 Kilohertz}

The calculation of variations in the coil impedance using the two sets of vector potential solutions $\left\{\boldsymbol{A}_{1}\right\}$ and $\left\{\boldsymbol{A}_{2}\right\}$ and the plotting of the impedance trajectory at the frequency of 10 kilohertz are discussed in the following. The graph presented in Figure 4 shows the absolute values of the per unit variations of the coil impedance components $\Delta r$ and $\Delta x$ in the range $-15.00 \leq d \leq 0.00$. Both characteristics represent approximations to unimodal functions, and the maximum variation of the coil impedance occurs when the coil displacement is $d=-3.25 \mathrm{~mm}$. The composition of these two characteristics in the complex $R$ - $X$ plane gives the impedance trajectory.

The relationship between the positional displacement $d$ of the eddy current coil and the variations $(\Delta r, \Delta x)$ that form the impedance trajectory is not clear at a first glance. In the attempt to clarify the subject, the $10 \mathrm{kHz}$ impedance trajectory is illustrated separately in Appendix B.

To introduce the basic operations that lead to the $10 \mathrm{kHz}$ impedance trajectory, let us initially consider the data presented in Table 2 . The data include ohmic and per unit values of the real and imaginary components of the coil impedance along two ranges 1 ) in the range $-4.00 \leq d \leq-3.00$ wherein the maximum variation in the coil impedance occurs. In the discussion, impedances $Z_{1}$ are related to the sequence of vector potentials $\left\{A_{1}\right\}$, whereas impedances $Z_{2}$ are related to the sequence of vector potentials $\left\{\boldsymbol{A}_{2}\right\}$; and 2 ) in the range $-15.00 \leq d \leq-14.00$ wherein the variations $\Delta r$ and $\Delta x$ tend to vanish. This can be observed on the last line of Table 2 . 
Table 2. Ohmic and per unit variations of the resistive and inductive components of the eddy current coil.

\begin{tabular}{cccccccccc}
\hline $\begin{array}{c}\text { Displacement } \\
(\mathrm{mm})\end{array}$ & $\begin{array}{c}\operatorname{Re}\left(Z_{1}\right) \\
(\Omega)\end{array}$ & $\begin{array}{c}\operatorname{Im}\left(Z_{1}\right) \\
(\Omega)\end{array}$ & $\begin{array}{c}\operatorname{Re}\left(Z_{2}\right) \\
(\Omega)\end{array}$ & $\begin{array}{c}\operatorname{Im}\left(Z_{2}\right) \\
(\Omega)\end{array}$ & $\begin{array}{c}\Delta R \\
(\Omega)\end{array}$ & $\begin{array}{c}\Delta X \\
(\Omega)\end{array}$ & $\begin{array}{c}\Delta Z \\
(\Omega)\end{array}$ & $\begin{array}{c}\Delta r \\
(\mathrm{pu})\end{array}$ & $\begin{array}{c}\Delta x \\
(\mathrm{pu})\end{array}$ \\
\hline-3.00 & 168.404 & 674.845 & 168.455 & 674.820 & -0.0510 & 0.025 & 0.0568 & -0.8909 & 0.4367 \\
-3.25 & 168.371 & 674.798 & 168.422 & 674.772 & -0.0510 & 0.026 & 0.0572 & -0.8909 & 0.4542 \\
-3.50 & 168.336 & 674.728 & 168.385 & 674.701 & -0.0490 & 0.027 & 0.0559 & -0.8559 & 0.4717 \\
-3.75 & 168.300 & 674.654 & 168.347 & 674.627 & -0.0470 & 0.027 & 0.0542 & -0.8210 & 0.4717 \\
-4.00 & 168.263 & 674.566 & 168.307 & 674.540 & -0.0440 & 0.026 & 0.0511 & -0.7686 & 0.4542 \\
$\ldots$ & $\ldots$ & $\ldots$ & $\ldots$ & $\ldots$ & $\ldots$ & $\ldots$ & $\ldots$ & $\ldots$ & $\ldots$ \\
-14.00 & 162.872 & 663.204 & 162.872 & 663.203 & 0.000 & 0.001 & 0.001 & 0.000 & 0.0175 \\
-14.25 & 162.760 & 663.054 & 162.760 & 663.054 & 0.000 & 0.000 & 0.000 & 0.000 & 0.0000 \\
-14.50 & 162.658 & 662.898 & 162.658 & 662.897 & 0.000 & 0.001 & 0.001 & 0.000 & 0.0175 \\
-14.75 & 162.572 & 662.808 & 162.572 & 662.807 & 0.000 & 0.001 & 0.001 & 0.000 & 0.0175 \\
-15.00 & 162.480 & 662.684 & 162.480 & 662.684 & 0.000 & 0.000 & 0.000 & 0.000 & 0.0000 \\
\hline
\end{tabular}

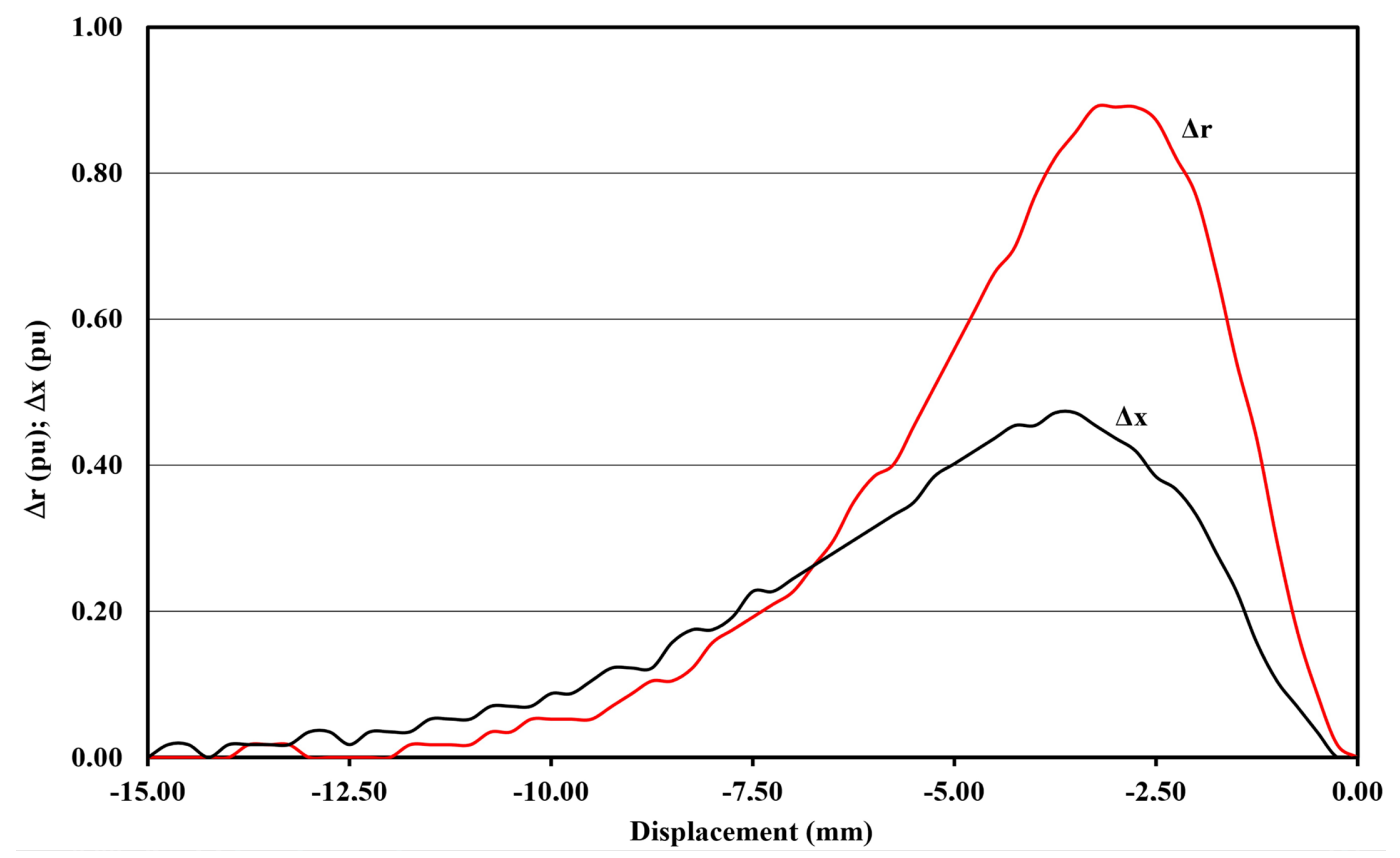

Figure 4. Absolute values of variations in the resistive and inductive components of the coil impedance at $10 \mathrm{kHz}$.

For a coil displacement $d=-3.25 \mathrm{~mm}$, the variation in magnitude of the coil impedance is maximum, $\Delta Z_{\max }=0.05724509 \Omega$, and this can be observed in line 2 and column 8 of Table 2 . To compute the ohmic variations of the coil impedance components $\Delta R$ and $\Delta X$ at this position, one may start by computing the variation of the resistive component $\Delta R$,

$$
\Delta R=\operatorname{Re}\left(Z_{1}\right)-\operatorname{Re}\left(Z_{2}\right)=-0.0510 \Omega .
$$

The variation of the inductive component $\Delta X$ is 


$$
\Delta X=\operatorname{Im}\left(Z_{1}\right)-\operatorname{Im}\left(Z_{2}\right)=-0.0260 \Omega .
$$

To compute the per unit variations of the components $\Delta r$ and $\Delta x$, their ohmic values should be normalized with respect to the maximum magnitude in variation of the coil impedance $\Delta Z_{\max }$ along the total excursion. For a coil displacement $d=-3.25 \mathrm{~mm}$, one has

$$
\Delta r=\Delta R / \Delta Z_{\max }=-0.8909 \text { p.u. }
$$

Also, at $d=-3.25 \mathrm{~mm}$, the per unit variation of the inductive component $\Delta x$ is

$$
\Delta x=\Delta X / \Delta Z_{\max }=0.4542 \text { p.u. }
$$

Values of the variables used in the calculations expressed by (1)-(4) appear highlighted on the $2^{\text {nd }}$ line of Table 2.

\section{Simulations at $\mathbf{1 0 0 . 0}$ Kilohertz}

For the operating frequency of 100 kilohertz, the graph presented in Figure 5 shows the per unit variations of the coil impedance components in the range $-11.75 \leq d \leq 0.00$.

It is worth noting that the characteristics shown in Figure 4 and Figure 5 are not directly comparable. Whereas at $10 \mathrm{kHz}$ the eddy current coil behaves as a predominantly resistive load, at $100 \mathrm{kHz}$ the eddy current coil behaves as a predominantly inductive load. For both components, $\Delta r$ and $\Delta x$, the coil scan carried out employing displacements of $0.25 \mathrm{~mm}$ has produced much smoother characteristics $\Delta r$ and $\Delta x$ for the operating frequency of $100 \mathrm{kHz}$.

The field simulator FEMM calculates the eddy current coil impedance, $Z$, as part of the "circuit properties" by using

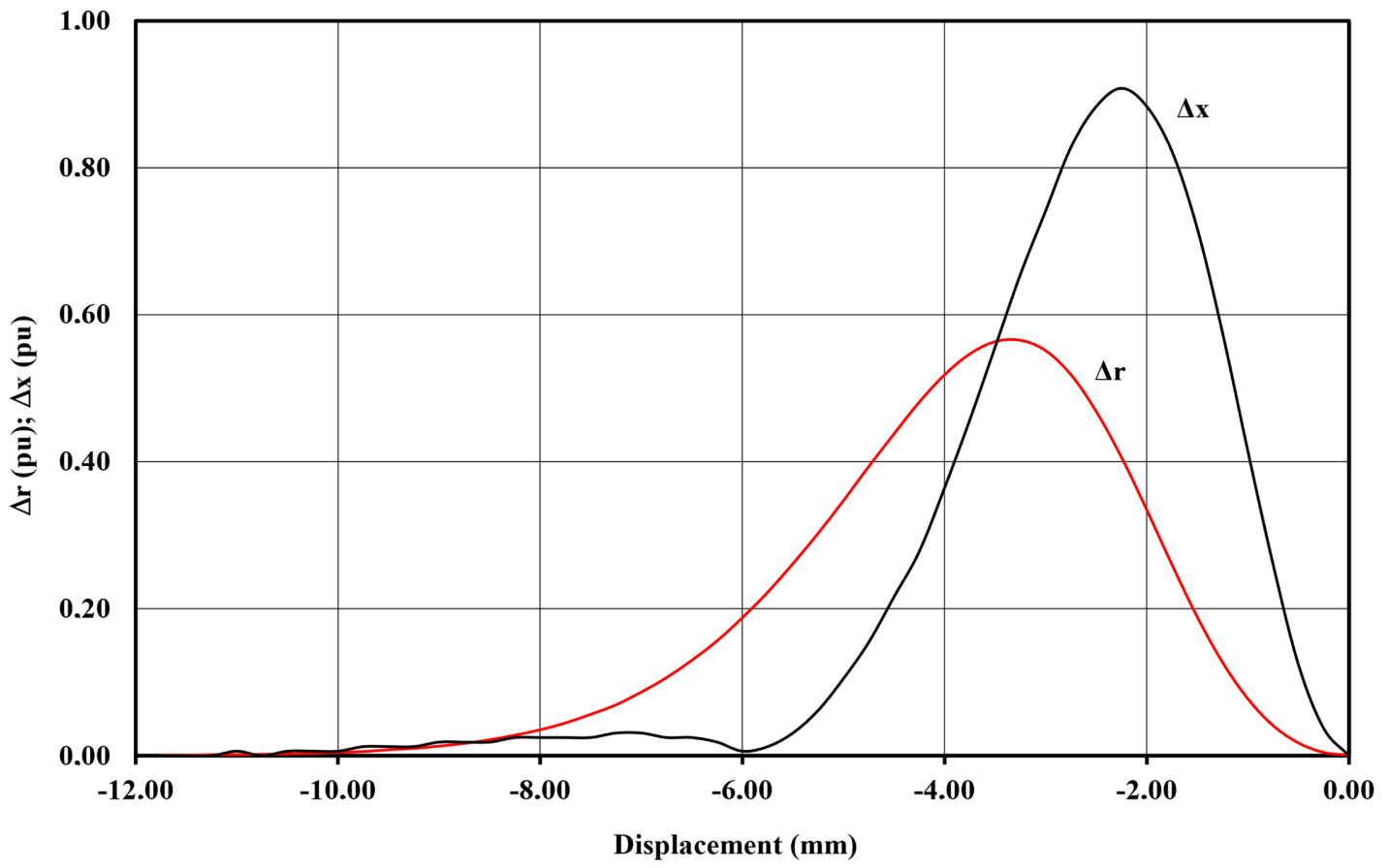

Figure 5. Variations in the resistive and inductive components of the coil impedance at $100 \mathrm{kHz}$. 


$$
Z=\boldsymbol{V}_{p} / \boldsymbol{I}_{p},
$$

where $V_{p}$ denotes the peak value of the terminal voltage, and $I_{p}$ denotes the peak value of the terminal current. To ensure that values of the coil impedance at different positions have been correctly computed, impedance values can also be computed using the alternative method explained in Appendix A.

To illustrate the use of the alternative method of impedance computation, let us consider the data presented in Table 3. The data include ohmic values of the impedance components $\operatorname{Re}_{1}(Z)$ and $\operatorname{Im}_{1}(Z)$ in the range $-3.00 \leq d \leq-2.00$. The maximum variation in the coil impedance is $\Delta Z_{\max }=1.61848 \Omega$ and occurs at $d$ $=-2.50 \mathrm{~mm}$. The data also include the coil dissipated energy $P$ and the magnetic stored energy $W$ in the domain of analysis. Now, let us consider the vector potential solution $A_{1}$ that represents the displacement $d=-2.50 \mathrm{~mm}$. For a "rms" current $I=10 \mathrm{~mA}$ and an angular frequency $\omega=2 \pi \times 1 \times 10^{5} \mathrm{rad} / \mathrm{s}$, the resistive component $R$ is given by

$$
R=P / I^{2}=530.076 \Omega,
$$

and the inductive reactance $X$ is given by

$$
X=(\omega 2 W) / I^{2}=6203.53 \Omega .
$$

Values calculated in (6) and (7) ought to be compared to the values indicated in columns 2 and 3 of the $3^{\text {rd }}$ line of Table 3 and calculated by the traditional field computation approach expressed by (5).

The composition, in the complex $R-X$ plane, of the two characteristics presented in Figure 5 produce the $100 \mathrm{kHz}$ impedance trajectory shown if Figure 6. This trajectory ought to be compared to the $10 \mathrm{kHz}$ impedance trajectory shown in Figure B3. It is worth noting that, as the distance between the detecting coil and the defect region increases, the variations $\Delta r$ and $\Delta x$ become smaller and more difficult to be computed accurately. The $10 \mathrm{kHz}$ impedance trajectory lacks smoothness, especially along its final portion that represents the coil scan below $d=-11.75 \mathrm{~mm}$. The reentrant corners present in the plot reflect the failure of the computation technique in detecting those very small variations in the resistive and inductive components of the eddy current coil. The $100 \mathrm{kHz}$ impedance trajectory, on the other hand, possesses the shape of a smooth convex characteristic, and the distribution of its discrete points is clearly in accordance with the physical understanding of the problem.

Table 3. Impedance, dissipated energy and the magnetic stored energy.

\begin{tabular}{cccccc}
\hline $\begin{array}{c}\text { Displacement } \\
(\mathrm{mm})\end{array}$ & $\begin{array}{c}\mathrm{Re}_{1}(Z) \\
(\Omega)\end{array}$ & $\begin{array}{c}\operatorname{Im}_{1}(Z) \\
(\Omega)\end{array}$ & $\begin{array}{c}\Delta Z \\
(\Omega)\end{array}$ & $\begin{array}{c}P \\
(\mathrm{~W})\end{array}$ & $\begin{array}{c}W \\
(\mathrm{~J})\end{array}$ \\
\hline-2.00 & 529.830 & 6203.81 & 1.5289 & 0.0529830 & $4.93666 \mathrm{E}-007$ \\
-2.25 & 529.930 & 6203.84 & 1.6101 & 0.0529930 & $4.93667 \mathrm{E}-007$ \\
-2.50 & 530.076 & 6203.75 & 1.6185 & 0.0530076 & $4.93661 \mathrm{E}-007$ \\
-2.75 & 530.156 & 6203.80 & 1.5810 & 0.0530156 & $4.93665 \mathrm{E}-007$ \\
-3.00 & 530.195 & 6203.72 & 1.4952 & 0.0530195 & $4.93658 \mathrm{E}-007$ \\
\hline
\end{tabular}




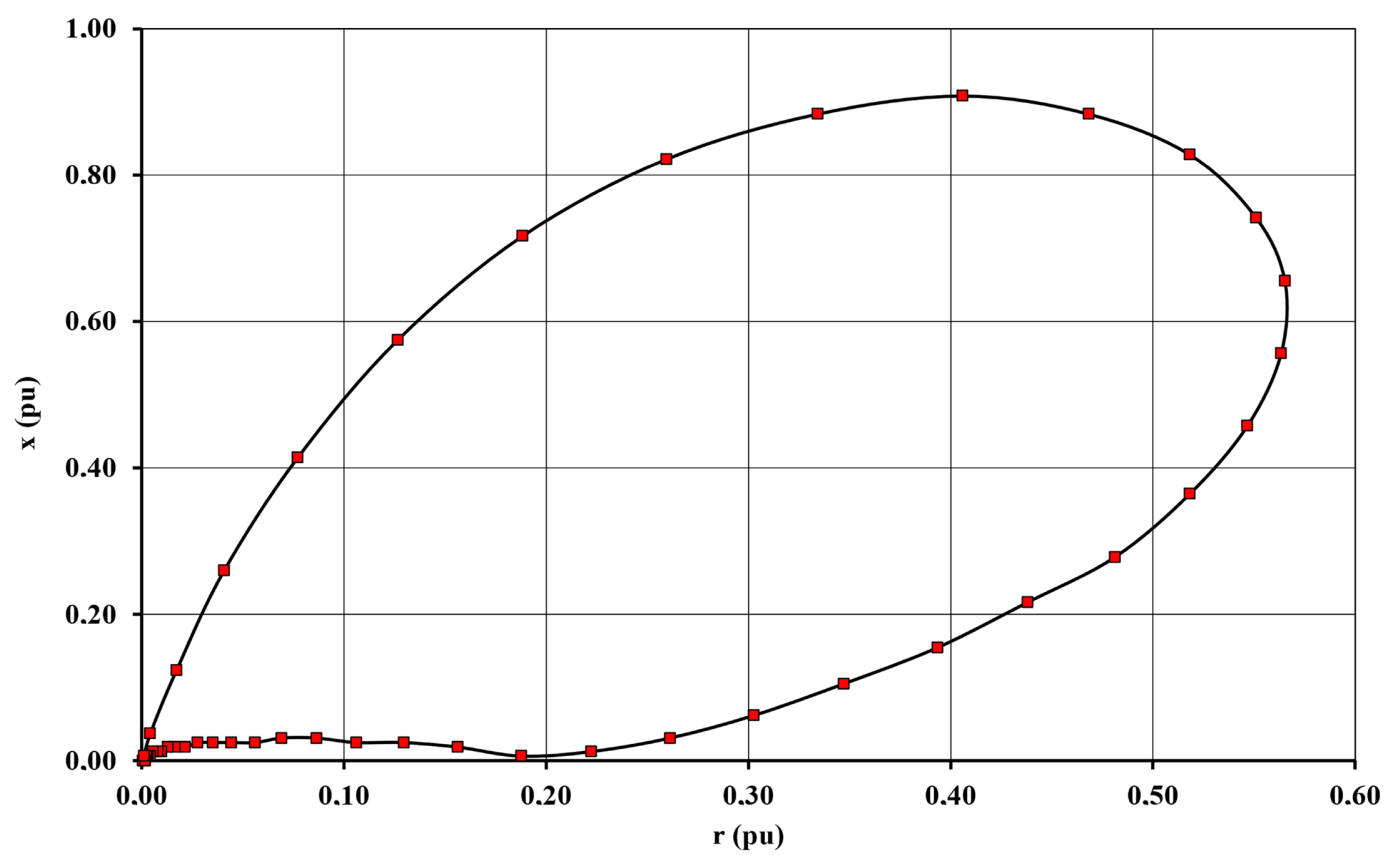

Figure 6. $100 \mathrm{kHz}$ impedance trajectory; coil displacement in the range $-11.75 \leq d \leq 0.00 \mathrm{~mm}$.

\section{Conclusions}

Eddy current testing (ECT) relies on the change in impedance of a detecting coil caused by the presence of electrical currents induced on a test specimen subjected to a time-varying magnetic field. The technique is used for the detection of cracks and other defects that interrupt the flow of the induced currents in the test specimen. The results are usually presented in the form of impedance trajectories in the complex $R-X$ plane. The technique does not depend on the magnetic properties of the material where the defect is located, and can be applied to any conducting material.

The WFNDEC's first eddy current benchmark problem can be viewed as a set of several ECT benchmark problems because it allows changes in geometry, material properties and frequency of excitation. The problem configuration selected for the simulated work includes the external support plate (SP), a pair of coils that accommodate 1000-not stranded copper wires, and a defect region symmetrically positioned around the origin of the $r$ - $z$ plane. The rms value of the excitation current is $10 \mathrm{~mA}$, and the analysis involves three different frequencies of excitation: 1,10 and $100 \mathrm{kHz}$.

The discussion places emphasis on the relationship between the positional displacement of the eddy current coil and the variations in the coil impedance used to obtain the impedance trajectory. For each of the three operating frequencies, numerical results include ohmic and per unit variations of the impedance components with respect to positional displacement and the resulting impedance trajectory.

For the operating frequency of $1 \mathrm{kHz}$, the ECT approach is clearly questionable for this class of problem, and the simulated experiment has failed in identi- 
fying impedance distortions along the coil scan. In the attempt to facilitate the teaching of ECT techniques on introductory courses on NDE, an additional effort has been made to better illustrate the $10 \mathrm{kHz}$ experiment. The most satisfactory results are associated to the frequency of $100 \mathrm{kHz}$.

Additional configurations of the WFNDEC' first problem remain to be tested in future work. Important investigations include 1) the system's response to higher frequencies of excitation; 2) further reduction in the area of the rectangular defect; and 3) changes in the geometry of the flaw considering both surface and under surface defects.

\section{Acknowledgements}

The authors acknowledge the financial support, in the form of scholarships, from the Brazilian Federal Agency for Postgraduate Studies (CAPES). The authors give thanks to David Meeker (dmeeker@ieee.org) for the use of the finite element CAD system.

\section{Conflicts of Interest}

The authors declare no conflicts of interest regarding the publication of this paper.

\section{References}

[1] Mihalache, O., Ueda, M. and Yamashita, T. (2007) 2D Axisymmetric ECT Simulation of the World Federation's First Eddy Current Benchmark Problem. In: Takahashi, S. and Kikuchi, H., Eds., Electromagnetic Nondestructive Evaluation $(X)$, Vol. 28, IOS Press, Amsterdam, 275-282.

[2] Meeker, D.C. (2018) Finite Element Method Magnetics, User's Manual, Version 4.2. http://www.femm.info/wiki/HomePage

[3] Ida, N. (1983) Three Dimensional Finite Element Modeling of Electromagnetic Nondestructive Testing Phenomena. Ph.D. Thesis, Colorado State University, Fort Collins.

[4] Liu, Z., Forsyth, D.S., Komorowski, J.P., Hanasaki, K. and Kirubarajan, T. (2007) Survey: State of the Art in NDE Data Fusion Techniques. IEEE Transactions on Instrumentation and Measurement, 56, 2435-2451. https://doi.org/10.1109/TIM.2007.908139

[5] Gros, X.E. (1997) A Review of NDT Data Fusion Processes and Applications. Insight, 39, 652-657. https://doi.org/10.1109/TIM.2007.908139

[6] Gros, A.X.E. (2000) Applications of NDT Data Fusion. Kluwer, Norwell.

[7] Brassard, O.M., Chehbaz, A., Pelletier, A. and Forsyth, D.S. (2000) Combined NDT Inspection Techniques for Corrosion Detection of Aircraft Structures. Proceedings of the 15th World Conference on Nondestructive Testing, Rome, 15-21 October 2000 .

[8] Deng, W., Matuszewski, B.J., Shark, L.K., Smith, J.P. and Cavaccini, G. (2004) Multi-Modality NDT Image Fusion and Its Mapping on Curved 3D CAD Surface. Proceedings of the 16th World Conference on Nondestructive Testing, Montreal, 30 August-3 September 2004.

[9] Sikora, R. and Palka, R. (2002) Review of Progress in Quantitative Nondestructive 
Evaluation, Vol. 21, 1909.

[10] Tian, Y., Li, Y., Zeng, Z., Udpa, L. and Udpa, S.S. (2004) Simulation of the World Federation's First Eddy Current Benchmark Problem. AIP Conference Proceedings, 700, 1560-1566. https://doi.org/10.1063/1.1711800

[11] Mihalache, O. (2006) Advanced Remote Field Computational Analysis of Steam Generators Tubes. Studies in Applied Electromagnetics and Mechanics, Electromagnetic Nondestructive Evaluation, 26, 220-227.

[12] (2005) FEMLAB Manual. COMSOL Inc., Stockholm.

[13] Nogueira, A.F.L., da Costa, V.H.P. and Weinert, R.L. (2017) Simulated Experiments for Teaching Mutually-Coupled Circuits CAD Techniques Using Analytic and Finite Element Solutions. Journal of Electromagnetic Analysis and Applications, 9, 183-202. https://doi.org/10.4236/jemaa.2017.911016

[14] Nogueira, A.F.L., Weinert, R.L. and Maldonado, L.J.A.S. (2019) Experiments for Teaching CAD Techniques Using Analytic and Finite Element Solutions of Electromagnetic Two-Dimensional Problems with Longitudinal Symmetry. Journal of Electromagnetic Analysis and Applications, 11, 79-99.

https://doi.org/10.4236/jemaa.2019.116006 


\section{Appendix A: Impedance Calculation in the $A-V$ Formulation}

Let $J_{e}$ denote the external current density on a conducting medium. One may define the electric field intensity $E$ by

$$
\boldsymbol{E}=-\nabla V-j \omega \boldsymbol{A},
$$

the magnetic flux density $B$ by

$$
B=\nabla \times A,
$$

and the magnetic field strength $H$ by

$$
\boldsymbol{H}=\frac{\boldsymbol{B}}{\mu},
$$

where $\omega$ is the angular frequency of the excitation. $V$ is the electric scalar potential, $A$ is the magnetic vector potential and $\mu$ is the magnetic permeability of the material medium. The impedance of the eddy current coil can be obtained by calculating the dissipated energy $P$ and the total magnetic stored energy $W$, using the following equations [3]:

$$
P=\int_{\Gamma} \boldsymbol{J}_{e} \boldsymbol{E}^{*} \mathrm{~d} \Gamma
$$

and

$$
W=\frac{1}{2} \int_{\Gamma} \boldsymbol{H} \boldsymbol{B}^{*} \mathrm{~d} \Gamma
$$

where $\Gamma$ refers to the whole solution domain, and $\left(^{*}\right)$ denotes the complex conjugate operator. The coil impedance is computed by

$$
R+j \omega L=\frac{1}{I^{2}}(P+j \omega 2 W),
$$

where $R$ is the resistance, $L$ is the inductance and $I$ is the "rms" current.

According to (Ida, 1983) [3], this method for calculating impedance based on the calculation of the dissipative and stored energy is always valid. In the case of axisymmetric configurations, the coil impedance can be calculated directly from the distribution of the magnetic vector potential $\boldsymbol{A}$ on the coil region (Tian et al., 2004) [10].

\section{Appendix B: Illustrations for the $10 \mathrm{kHz}$ Impedance Trajectory}

In the following, the $10 \mathrm{kHz}$ impedance trajectory is presented as the union of two plots 1) the first plot represents the portion of the impedance trajectory obtained when the coil displacement $d$ varies along the initial $1 / 3$ of the total excursion, i.e., $-5.0 \leq d \leq 0.0 \mathrm{~mm}$; and 2) the second plot represents the portion of the impedance trajectory obtained when the coil displacement $d$ varies along the remaining $2 / 3$ of the total excursion, i.e., $-15.00 \leq d \leq-5.00 \mathrm{~mm}$. The first plot is presented in Figure B1, the second plot is presented in Figure B2, and the final plot, representing the complete impedance trajectory is presented in Figure B3. 


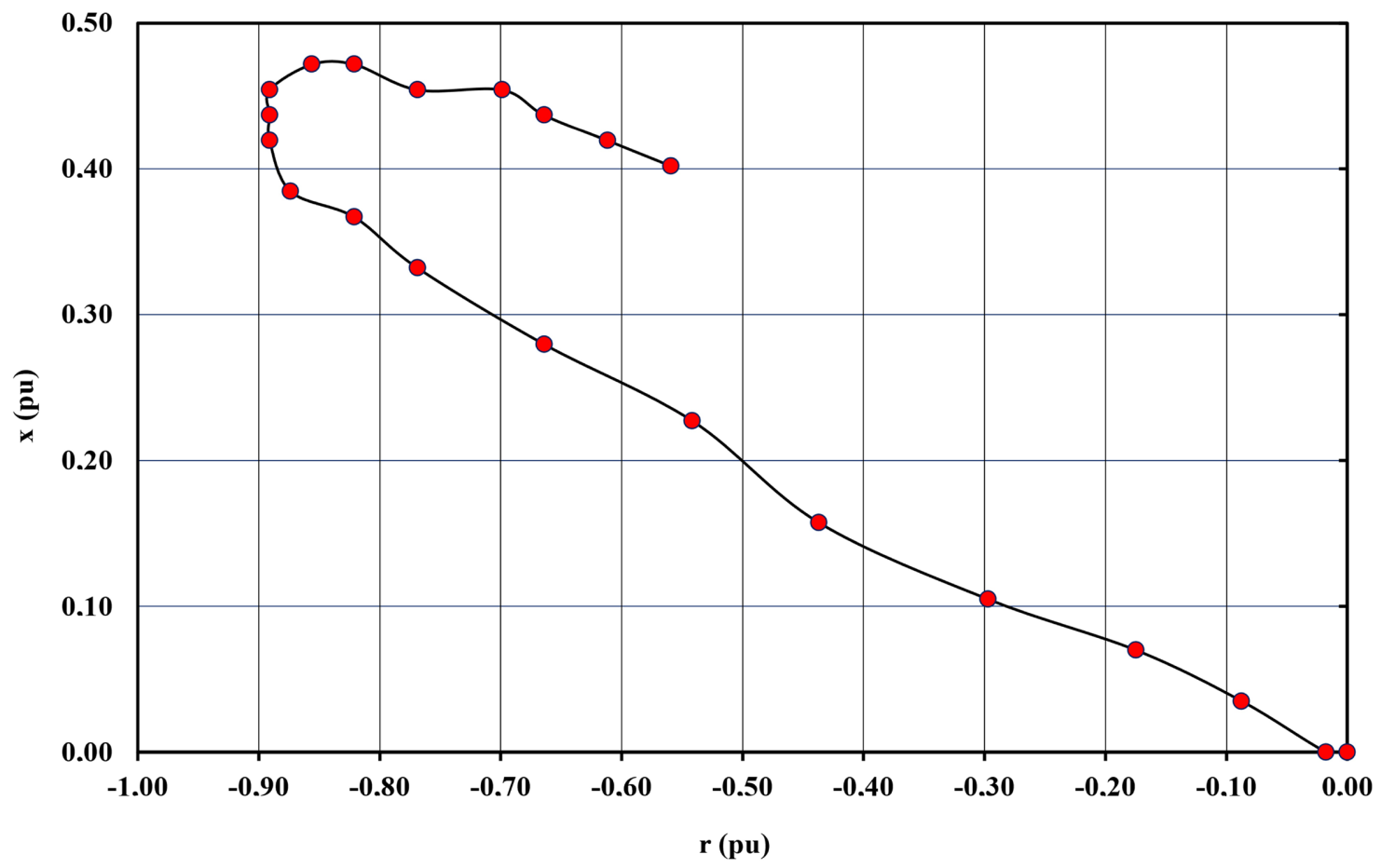

Figure B1. Initial portion of the $10 \mathrm{kHz}$ impedance trajectory; $-5.0 \leq d \leq 0.00 \mathrm{~mm}$.

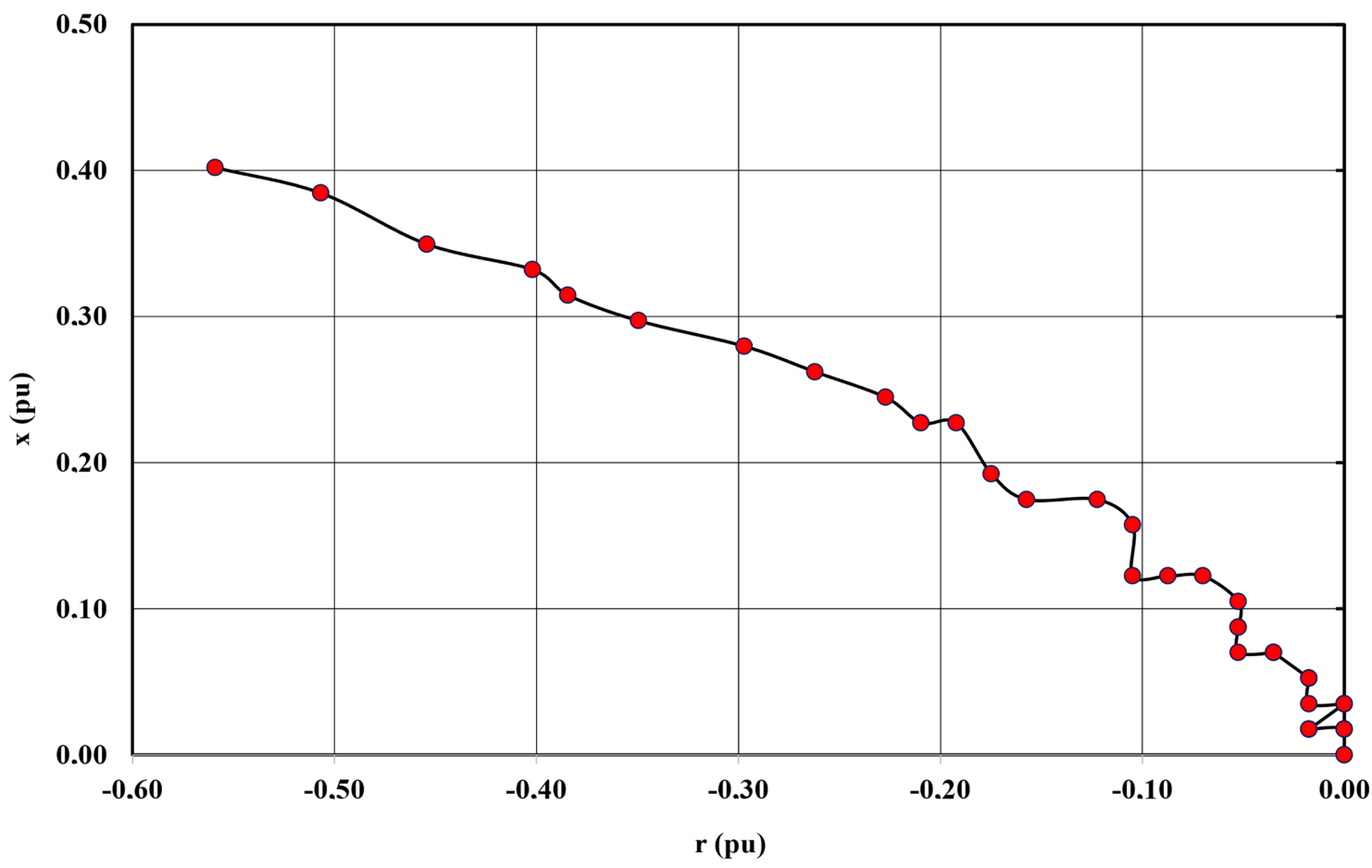

Figure B2. Second portion of the $10 \mathrm{kHz}$ impedance trajectory; $-15.0 \leq d \leq-5.0 \mathrm{~mm}$. 


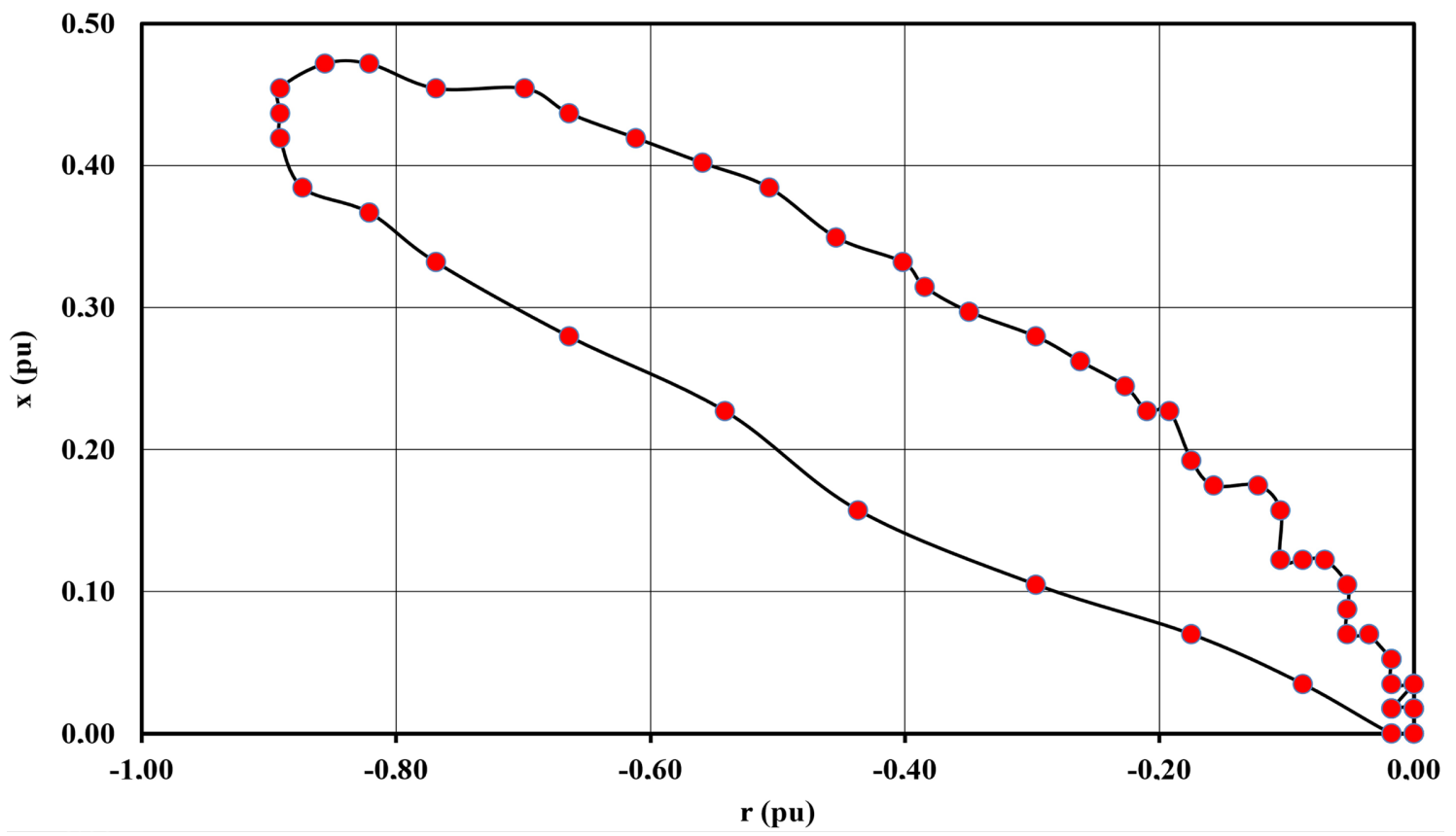

Figure B3. $10 \mathrm{kHz}$ impedance trajectory; $-15.0 \leq d \leq 0.0 \mathrm{~mm}$. 